\title{
Hydromagnetic Non-Darcian Free-Convective Flow of a Non-Newtonian Fluid with Temperature Jump
}

\author{
Ahmed M. Salem ${ }^{1,2}$ and Mohamed Abd El-Aziz ${ }^{2,3}$ \\ ${ }^{1}$ Mathematics Department, Faculty of Science and Arts, King Khalid University, Belqarn, \\ P.O. Box 61985, Saudi Arabia \\ ${ }^{2}$ Mathematics Department, Faculty of Science, Helwan University, P.O. Box 11795, Cairo, Egypt \\ ${ }^{3}$ Mathematics Department, Faculty of Science, King Khalid University, Abha, P.O. Box 9004, Saudi Arabia \\ Correspondence should be addressed to Ahmed M. Salem; ah_marei@hotmail.com
}

Received 16 February 2013; Accepted 30 March 2013

Academic Editor: Mohamed Seddeek

Copyright (C) 2013 A. M. Salem and M. Abd El-Aziz. This is an open access article distributed under the Creative Commons Attribution License, which permits unrestricted use, distribution, and reproduction in any medium, provided the original work is properly cited.

\begin{abstract}
In the present study, the effect of viscous dissipation on magnetohydrodynamic (MHD) non-Darcian free-convection flow of a non-Newtonian power-law fluid past a vertical flat plate in a saturated porous medium with variable viscosity and temperature jump is considered. The fluid is permeated by a transverse magnetic field imposed perpendicularly to the plate on the assumption of a small magnetic Reynolds number. The fluid viscosity is assumed to vary as a reciprocal of linear function of temperature. The governing boundary layer equations and boundary conditions are cast into a dimensionless form and simplified by using a similarity transformation into a system of nonlinear ordinary differential equations and solved numerically. The effects of the governing parameters on the flow fields and heat transfer are shown in graphs and tabular form.
\end{abstract}

\section{Introduction}

The analysis of convective heat transfer over a vertical plate in a non-Newtonian fluid saturated porous medium is important for the thermal design of industrial equipment dealing with molten plastics, polymeric liquids, oil extraction, ground water pollution, thermal insulation, solid matrix heat exchangers, the storage of nuclear waste, packed-bed catalytic reactors, foodstuffs and slurries, and so forth. Thus, considerable efforts have been directed toward major aspects of this coupled, nonlinear boundary-layer problem. Most of the earlier studies were based on Darcy's law which states that the volume-averaged velocity is proportional to the pressure gradient. The Darcy model is shown to be valid under the conditions of low velocities and small porosity, Cheng and Hsu [1]. In many practical situations, the porous medium has higher flow rates and reveals nonuniform porosity distribution in the near wall region, making the Darcy's law inapplicable. To model the real physical situation better, it is therefore necessary to include the aforementioned nonDarcian terms in the analysis of convective transport in a porous medium. The inertia effect is expected to be important at a higher flow rate, and it can be accounted for through the addition of a velocity-squared term in the momentum equation, which is known as the Forchheimer extension. The boundary effect may become significant when heat transfer is considered in a region very close to a solid boundary. The Brinkman's extension, which incorporates a viscous shear stress term into the momentum equation, together with the no-slip boundary condition, is usually used to shed light on the importance of boundary effects. A comment was made by Fand and Brucker [2] that the effect of viscous dissipation might be significant in the case of natural convection in porous medium in connection with their experimental correlation for heat transfer in external flows. The validity of the comment was tested for the Darcy model by Fand et al. [3], both experimentally and analytically while estimating the heat transfer coefficient from a horizontal cylinder embedded in a saturated porous medium. Their mathematical analysis is confined to studying the dissipation effect using a steady, $1 \mathrm{D}$ energy equation; the basis of the equation is from the analogy given by Bejan [4] for the inclusion of viscous 
dissipation effects. The influence of viscous dissipation can be seen from the analogy given by Tucker and Dessenberger [5] to model the heat transfer and fluid flow through porous media in order to study the resin transfer molding (R.T.M.) for producing fiber-reinforced polymeric parts in final shape. Ibrahim et al. [6] studied the problem of natural convective heat transfer for a non-Newtonian fluid from an impermeable vertical plate embedded in a fluid-saturated porous medium. El-Amin [7] investigated the influences of viscous dissipation on buoyancy-induced flow over (a horizontal or a vertical flat plate) embedded in a non-Newtonian fluid-saturated porous medium. Mansour and El-Shaer [8] studied the effect of magnetic field on non-Darcy axisymmetric free convection in a power-law fluid-saturated porous medium with variable permeability. Mahdy [9] studied the flow, heat, and mass transfer characteristics of non-Darcian mixed convection of a non-Newtonian fluid from a vertical isothermal plate embedded in a homogeneous porous medium with the effect of Soret and Dufour. It is known that the fluid properties change with temperature especially the fluid viscosity. So in order to predict accurately the flow behavior, it is necessary to take into account this variation of viscosity since the recent results on the flow of non-Newtonian fluid have shown that when this effect is included, the flow characteristics may be substantially changed compared to the constant viscosity case. The effect of variable viscosity on non-Darcy free or mixed convective heat transfer along a vertical surface embedded in a porous medium saturated with a nonNewtonian fluid is analysed by Jayanthi and Kumari [10]. Salem [11] studied the problem of flow and heat transfer of an electrically conducting viscoelastic fluid over a continuously stretching sheet in the presence of a uniform magnetic field.

The present work is devoted to study the problem of freeconvective heat transfer from a vertical flat plate embedded in a non-Darcian porous medium saturated with a power law non-Newtonian fluid. The fluid is subjected to a transverse uniform magnetic field. It is assumed that the magnetic Reynolds number is small so that the induced magnetic field is neglected. Viscous dissipation due to mechanical work is included in the temperature field. The fluid viscosity is assumed to vary as a reciprocal of linear function of temperature. Temperature jump is also considered which gives interesting features regarding such flow.

\section{Analysis}

Let us consider the flow of steady, laminar, incompressible, two-dimensional, non-Darcy, non-Newtonian powerlaw fluid flow with heat transfer along a vertical flat plate in a saturated porous medium. It is assumed that the fluid and the solid matrix are everywhere in local thermal equilibrium. The $x$-axis is directed along the plate and points to the direction of motion. The $y$-axis is perpendicular to the plate. The fluid is permeated by an imposed uniform magnetic field of strength $B_{0}$, which acts in the positive $y$-axis. The magnetic Reynolds number is taken to be small enough so that the induced magnetic field can be neglected. With these assumptions and the application of the Boussinesq and boundary layer approximations, the governing equations, namely, the equation of continuity, the non-Darcy flow model (i.e., the model given by Shenoy [12]), and the energy equation, are

$$
\begin{gathered}
\frac{\partial u}{\partial x}+\frac{\partial v}{\partial y}=0 \\
\frac{\partial}{\partial y}\left(\mu u^{n}\right)+\rho b k^{*} \frac{\partial}{\partial y}\left(u^{2}\right)=k^{*}\left(\rho g \beta \frac{\partial T}{\partial y}-\sigma B_{0}^{2} \frac{\partial u}{\partial y}\right) \\
u \frac{\partial T}{\partial x}+v \frac{\partial T}{\partial y}=\alpha \frac{\partial^{2} T}{\partial y^{2}}+\frac{\mu}{k^{*} \rho c_{p}}\left(u^{n-1}+\frac{\rho b k^{*}}{\mu} u\right) u^{2}
\end{gathered}
$$

The associated boundary conditions are

$$
\begin{aligned}
& v=0, \quad T=T_{w}+N \frac{\partial T}{\partial y} \quad \text { at } y=0, \\
& u \longrightarrow 0, \quad T \longrightarrow T_{\infty} \quad \text { as } y \longrightarrow \infty .
\end{aligned}
$$

Here $x, y$ are the Cartesian coordinates, $u$ and $v$ the velocity components, $n$ the power-law index, $\mu$ the viscosity, $T$ the temperature, $\alpha$ the effective thermal diffusivity of the saturated porous medium, $\beta$ the volumetric thermal expansion, $\sigma$ the electric conductivity, $b$ an empirical constant associated with the Forchheimer inertia term, $\rho$ the density, $c_{p}$ the specific heat at constant pressure, $g$ the acceleration due to gravity, $k^{*}$ is the modified permeability for the flow of non-Newtonian power law fluid, and $N$ is the thermal slip factor. Christopher and Middleman [13] and Dharmadhikari and Kale [14] proposed the following relationships for the permeability $k^{*}$ as a function of the power-law index $n$ :

$$
k^{*}=\left(\frac{6}{25}\right)\left(\frac{n \varepsilon}{3 n+1}\right)^{n}\left(\frac{\varepsilon d}{3(1-\varepsilon)}\right)^{n+1}
$$

(see Christopher and Middleman [13]),

$$
k^{*}=\frac{6}{\varepsilon}\left(\frac{6 n+1}{10 n+3}\right)^{n}\left(\frac{75}{16}\right)^{3(10 n-3) /(10 n+11)}\left(\frac{\varepsilon^{2} d}{8(1-\varepsilon)}\right)^{n+1}
$$

(see Dharmadhikari and Kale [14]).

In the above equation, $\varepsilon$ is the porosity and $d$ is the pore diameter.

The fluid properties are assumed to be isotropic and constant, except for the fluid viscosity which is assumed to be reciprocal of a linear function of temperature and can be written as (Kumari [15], Lai and Kulacki [16])

$$
\mu=\mu_{\infty}\left(\frac{1}{1+\gamma\left(T-T_{\infty}\right)}\right)
$$

or

$$
\mu=\frac{1}{a\left(T-T_{\infty}\right)},
$$

where

$$
a=\frac{\gamma}{\mu_{\infty}}, \quad T_{r}=T_{\infty}-\frac{1}{\gamma}
$$


In the above relations, both $a$ and $T_{r}$ are constants, and their values depend on the reference state and the thermal property of the fluid $\gamma$. The viscosity of a liquid usually decreases with increasing temperature and it increases with gases in general, $a>0$ for liquids and $a<0$ for gases.

To proceed, we define the dimensionless variables

$$
\begin{gathered}
\eta=\frac{y}{x} \sqrt{\mathrm{Ra}_{x}}, \quad \psi=\alpha \sqrt{\mathrm{Ra}_{x}} f(\eta), \\
\theta(\eta)=\frac{T-T_{r}}{T_{w}-T_{\infty}}+\theta_{r},
\end{gathered}
$$

where $\operatorname{Ra}_{x}=(x / \alpha)\left(k^{*} \rho g \beta\left(T_{w}-T_{\infty}\right) / \mu_{\infty}\right)^{1 / n}$ is the local rayleigh number, $\psi$ is the physical stream function defined so that $u=\partial \psi / \partial y, v=-\partial \psi / \partial x$, and the continuity equation is satisfied automatically, $\theta_{r}=\left(T_{r}-T_{\infty}\right) /\left(T_{w}-T_{\infty}\right)$ is the viscosity/temperature parameter, $f$ is a dimensionless stream function, $\eta$ is a similarity space variable, and $\theta$ is the dimensionless temperature. The velocity components are readily obtained as

$$
u=\frac{\alpha}{x} \operatorname{Ra}_{x} f^{\prime}, \quad v=-\frac{\alpha}{2 x} \sqrt{\mathrm{Ra}_{x}}\left(f-\eta f^{\prime}\right) .
$$

The mathematical problem defined in (2) and (3) is then transformed into the coupled nonlinear ordinary differential equations and their associated boundary conditions:

$$
\begin{gathered}
\frac{\theta_{r}}{\left(\theta_{r}-\theta\right)^{2}}\left(f^{\prime}\right)^{n} \theta^{\prime}+\frac{n \theta_{r}}{\left(\theta_{r}-\theta\right)}\left(f^{\prime}\right)^{n-1} f^{\prime \prime} \\
+2 G r^{*} f^{\prime} f^{\prime \prime}=\theta^{\prime}-M f^{\prime \prime}, \\
\theta^{\prime \prime}+\mathrm{Ec} f^{\prime}\left[\frac{\theta}{\theta_{r}-\theta}\left(f^{\prime}\right)^{n}+\mathrm{Gr}^{*}\left(f^{\prime \prime}\right)^{2}\right]=-\frac{1}{2} f \theta^{\prime}, \\
f(0)=0, \quad \theta(0)=1+\Lambda \theta^{\prime}(0), \\
f^{\prime}(\infty)=0, \quad \theta(\infty)=0,
\end{gathered}
$$

where prime denotes ordinary differentiation with respect to $\eta, \mathrm{Ra}_{x}$ is the local rayleigh number, $M=\left(\sigma B_{0}^{2} k^{*} / \mu_{\infty} \mathrm{Ra}_{x}^{n-1}\right)(x / \alpha)^{n-1}$ is the magnetic field parameter, $\mathrm{Gr}^{*}=f_{0} \mathrm{Ra}_{d}^{2-n}$ is the non-Darcian parameter with $f_{0}=(\alpha / d)^{2-n}\left(\rho b k^{*} / \mu_{\infty}\right)$ representing the structural and thermophysical properties of the porous medium and $\mathrm{Ra}_{d}$ the pore diameter-dependent Rayleigh number, which describes the relative intensity of the buoyancy force and the dispersion parameter, $\mathrm{Ec}=x g \beta / c_{p}$ is the Eckert number, and $\Lambda=N_{0}(a / \alpha)^{1 / 2}$ is the temperature jump parameter.

The physical quantity of interest is the local Nusselt number $\mathrm{Nu}_{x}$ which is defined by

$$
\mathrm{Nu}_{x}=-\frac{x}{T_{w}-T_{\infty}}\left(\frac{\partial T}{\partial y}\right)_{y=0}=-\mathrm{Ra}_{x}^{1 / 2} \theta^{\prime}(0) .
$$

\section{Numerical Solution}

The coupled non-linear differential equations (12) and (13) subject to the boundary conditions (14) and (15) constitute a two-point boundary value problem. In order to solve these equations numerically, we follow the most efficient numerical shooting technique with fifth-order Runge-Kutta-Fehlberg integration scheme. In this method, it is most important to choose the appropriate finite values of $\eta \rightarrow \infty$. To select $\eta_{\infty}$, we begin with some initial guess value and solve the problem with some particular set of parameters to obtain $f^{\prime}(0)$ and $\theta^{\prime}(0)$. The solution process is repeated with another large value of $\eta_{\infty}$ until two successive values of $f^{\prime}(0)$ and $\theta^{\prime}(0)$ differ only after desired digit signifying the limit of the boundary along $\eta$. The last value of $\eta_{\infty}$ is chosen as appropriate value of the limit $\eta \rightarrow \infty$ for that particular set of parameters. Equations (12) and (13) were first formulated as a set of four first-order simultaneous equations of four unknowns following the method of superposition [17]. Thus, we set $y_{1}=f, y_{2}=f^{\prime}, y_{3}=\theta$, and $y_{4}=\theta^{\prime}$; (12) and (13) then reduced into a system of ordinary differential equations; that is,

$$
\begin{gathered}
y_{1}^{\prime}=y_{2}, \quad y_{1}(0)=0 \\
y_{2}^{\prime}=\frac{y_{4}\left[\left(\theta_{r}-y_{3}\right)^{2}-\theta_{r} y_{2}^{n}\right]}{\left[n \theta_{r}\left(\theta_{r}-y_{3}\right) y_{2}^{n-1}+\left(\theta_{r}-y_{3}\right)^{2}\left(2 \mathrm{Gr}^{*} y_{2}+M\right)\right]}, \\
y_{2}(0)=\delta_{1}, \\
y_{3}^{\prime}=y_{4}, \quad(0)=1+\Lambda \delta_{2}, \\
y_{4}^{\prime}=-\frac{1}{2} y_{1} y_{4}-\operatorname{Ec}\left(\frac{\theta_{r}}{\left(\theta_{r}-y_{3}\right)} y_{2}^{n+1}+\mathrm{Gr} y_{2}^{3}\right) \\
y_{4}(0)=\delta_{2},
\end{gathered}
$$

where $\delta_{1}$ and $\delta_{2}$ are determined such that they satisfy $y_{2}(\infty)=0$ and $y_{4}(\infty)=0$. The shooting method is used to guess $\delta_{1}$ and $\delta_{2}$ until the boundary conditions $y_{2}(\infty)=0$ and $y_{4}(\infty)=0$ are satisfied. Then the resulting differential equations can be integrated by fifth-order Runge-Kutta-Fehlberg integration scheme. The above procedure is repeated until we get the results up to the desired degree of accuracy, $10^{-6}$.

\section{Results and Discussion}

The system of transformed governing equations (12) and (13) subject to the boundary conditions (14) and (15) is solved numerically using the shooting method. Solutions are obtained for both Darcy $\left(\mathrm{Gr}^{*}=0\right)$ and non-Darcy $\left(\mathrm{Gr}^{*}=\right.$ 2) cases and different values of power-law index $n(0.5 \leq$ $n \leq 1.5)$, magnetic field parameter $M(0 \leq M \leq 2)$, viscosity/temperature parameter $\theta_{r}\left(-\infty \leq \theta_{r} \leq-1\right)$, viscous dissipation parameter (the Eckert number) $\mathrm{Ec}(0 \leq \mathrm{Ec} \leq 0.8)$, and temperature jump parameter $\Lambda(0 \leq \Lambda \leq 0.8)$.

Table 1 shows a comparison of the present results for $-\theta^{\prime}(0)$ with those reported by Ibrahim et al. [6] for various values of $n$ and $\mathrm{Gr}^{*}$ in the absence of magnetic parameter $(M=0)$, temperature jump $(\Lambda=0)$, viscous dissipation $($ Ec $=0)$, and variable viscosity $\left(\theta_{r} \rightarrow-\infty\right)$. It can be seen 


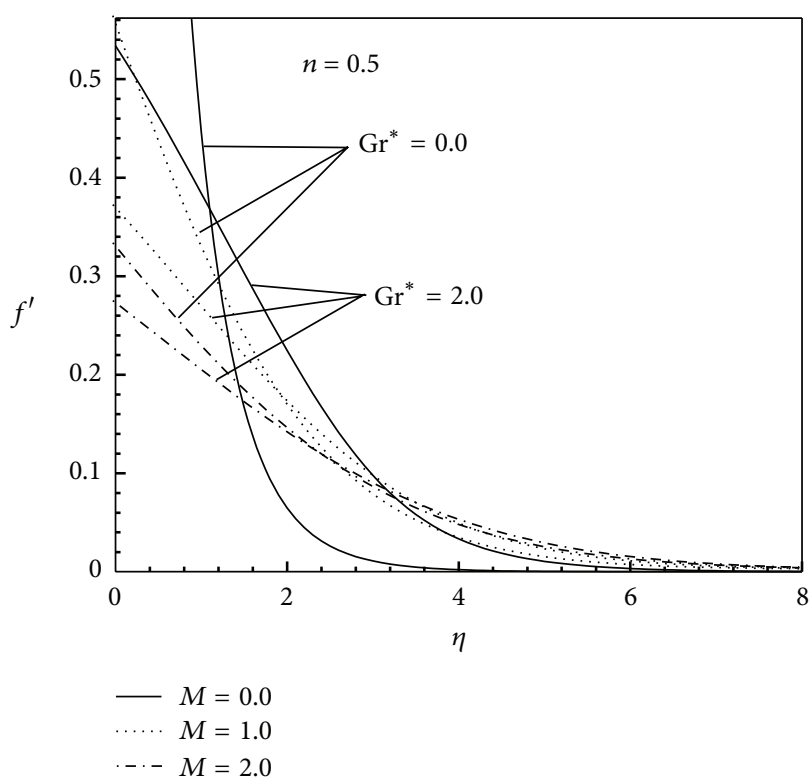

(a)

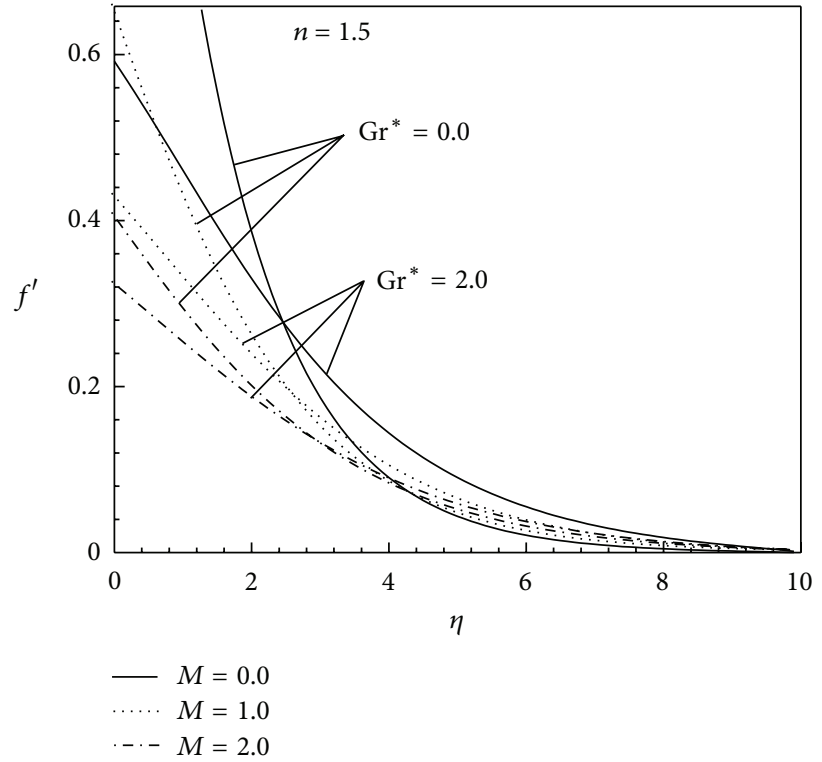

(b)

Figure 1: (a) Velocity profiles in the Darcy and non-Darcy cases for various values of $M$ with $n=0.5, \theta_{r}=-1.0, \Lambda=0.2$, and Ec $=0.05$. (b) Velocity profiles in the Darcy and non-Darcy cases for various values of $M$ with $n=1.5, \theta_{r}=-1.0, \Lambda=0.2$, and Ec $=0.05$.

TABLE 1: Comparison of the values of $-\theta^{\prime}(0)$ for $\Lambda=\mathrm{Ec}=M=0$, $\theta_{r} \rightarrow-\infty$ and various values of $n$ and $\mathrm{Gr}^{*}$ with previously published data.

\begin{tabular}{lccc}
\hline$n$ & Gr* & $\begin{array}{c}-\theta^{\prime}(0) \\
{[6]}\end{array}$ & Present results \\
\hline 0.5 & 0.0 & 0.40613 & 0.399325 \\
0.5 & 1.0 & 0.32263 & 0.319624 \\
1.0 & 0.0 & 0.44455 & 0.444552 \\
1.0 & 1.0 & 0.38064 & 0.379509 \\
\hline
\end{tabular}

from this table that the comparison is excellent especially for higher values of the viscosity index $(n=1)$ and smaller values of the non-Darcian parameter $\left(\mathrm{Gr}^{*}=0\right)$.

Typical velocity distributions are presented at selected values of $M$ and $\mathrm{Gr}^{*}$ for a shear thinning fluid of $n=0.5$ in Figure 1(a) and for a shear thickening fluid of $n=1.5$ in Figure 1(b) when $\theta_{r}=-1, \Lambda=0.2$, and $\mathrm{Ec}=0.05$. It is seen from these figures that the magnetic field decreases the velocity $f^{\prime}$ in both Darcy $\left(\mathrm{Gr}^{*}=0\right)$ and non-Darcy $\left(\mathrm{Gr}^{*}=2\right)$ flow regimes, and this behavior is more noticeable for the Darcy flow case. Also, the momentum boundary layer thickness for shear thickening fluid is larger than that for shear-thinning fluid. Moreover, for the case of Darcy flow $\left(\mathrm{Gr}^{*}=0\right)$, the velocity decreases dramatically in the absence of the magnetic field $M$, but this behavior is more prominent for a shear thinning fluid.

Representative temperature $\theta(\eta)$ profiles within the boundary layer for the same values of parameters considered in Figures 1(a) and 1(b) are presented in Figure 2(a) for a shear thinning fluid of $n=0.5$ and in Figure 2(b) for a shear thickening fluid of $n=1.5$. For both Darcy and nonDarcy flow regimes, it is clear from these figures that the temperature $\theta(\eta)$ increases with an increase in the thermal boundary layer thickness as the magnetic field $M$ is increased. Further, Figure 2(a) shows that the effect of $M$ in the nonDarcy flow case is seen to be more pronounced for the shear thinning fluid, while the opposite result is observed when the fluid is shear thickening fluid as observed from Figure 2(b). For given $M$, Figure 2(b) shows that the effect of non-Darcian parameter $\mathrm{Gr}^{*}$ is to increase the temperature $\theta(\eta)$ for the case of a shear thickening fluid, while the opposite behavior is obtained when the fluid is shear thinning as shown in Figure 2(a).

Variations of the dimensionless velocity profiles $f^{\prime}(\eta)$ for various values of the Eckert number Ec and non-Darcian parameter $\mathrm{Gr}^{*}$ are shown in Figure 3(a) for $n=0.5$ (shear thinning fluid) and in Figure 3(b) for $n=1.5$ (shear thickening fluid), while the corresponding temperature $\theta(\eta)$ profiles are presented in Figure 4(a) for $n=0.5$ and in Figure 4(b) for $n=1.5$. It is clear from these figures that as compared to the case of no viscous dissipation $(\mathrm{Ec}=0)$, it can be seen that the dimensionless velocity and temperature are increased with increasing the viscous dissipation Ec. This phenomenon is evident from the fact that viscous dissipation produces heat due to drag between the fluid particles, and this extra heat causes an increase in the fluid initial temperature, which in turn gives rise to the velocity of the fluid particles, and hence the fluid velocity increases. It is also observed that a distinctive peak in the temperature profile occurs in the fluid adjacent to the plate for higher values of $\mathrm{Ec}(\mathrm{Ec}=$ 0.8 ). The presence of the peak implies that the temperature attains maximum value inside the body of the fluid adjacent to the surface but not at the surface, and therefore heat is 


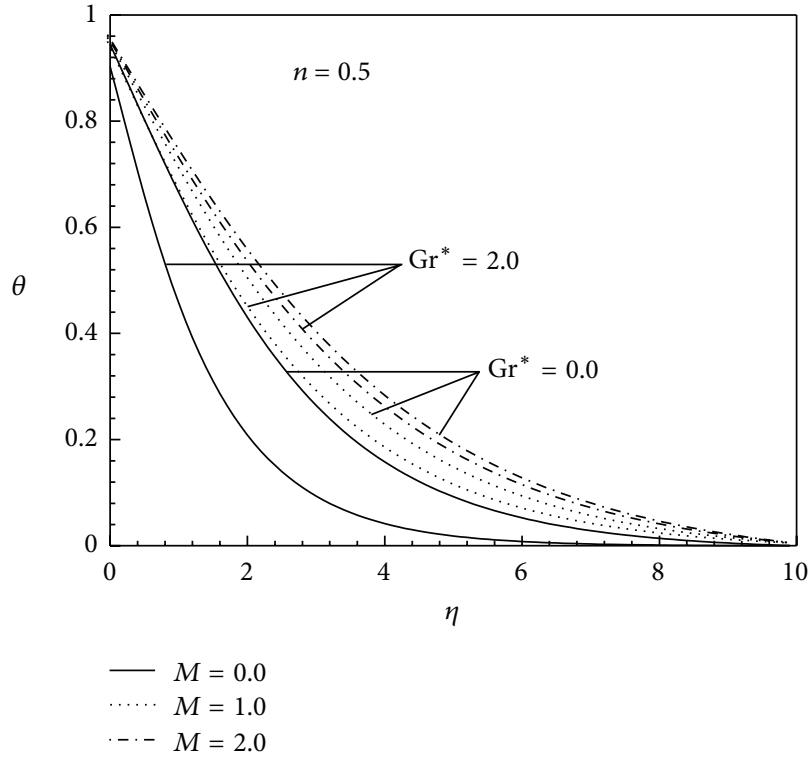

(a)

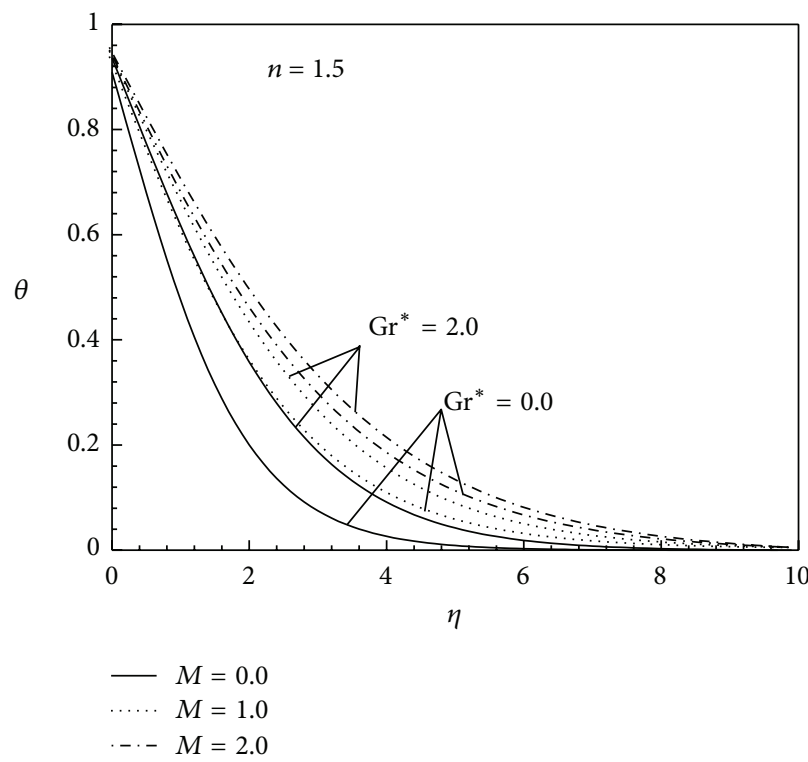

(b)

Figure 2: (a) Temperature profiles in the Darcy and non-Darcy cases for various values of $M$ with $n=0.5, \theta_{r}=-1.0, \Lambda=0.2$, and Ec $=0.05$. (b) Temperature profiles in the Darcy and non-Darcy cases for various values of $M$ with $n=1.5, \theta_{r}=-1.0, \Lambda=0.2$, and Ec $=0.05$.

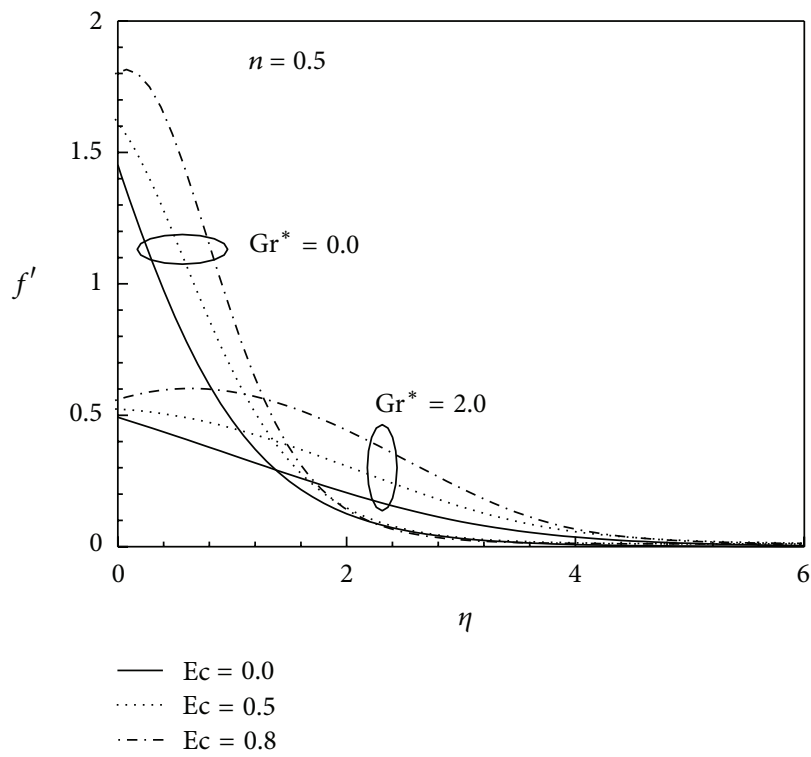

(a)

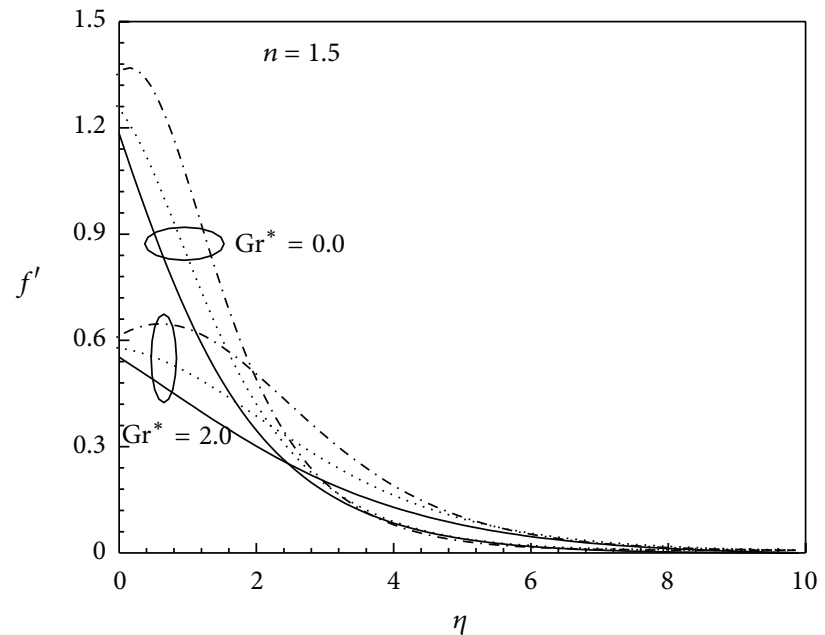

$\begin{aligned}- & \mathrm{Ec}=0.0 \\ \ldots . & \mathrm{Ec}=0.5 \\ \ldots . & \mathrm{Ec}=0.8\end{aligned}$

FIGURE 3: (a) Velocity profiles in the Darcy and non-Darcy cases for various values of Ec with $n=0.5, \theta_{r}=-1.0, M=0.2$, and $\Lambda=0.2$. (b) Velocity profiles in the Darcy and non-Darcy cases for various values of Ec with $n=1.5, \theta_{r}=-1.0, M=0.2$, and $\Lambda=0.2$.

expected to transfer from the ambient fluid to the surface. This behavior is true in the Darcy $\left(\mathrm{Gr}^{*}=0\right)$ and non-Darcy $\left(\mathrm{Gr}^{*}=2\right)$ flow cases. Also, the increase in the fluid velocity and temperature due to viscous heating is observed to be more pronounced for a higher value of Ec, as expected. Further, viscous dissipation demonstrates a more pronounced influence on the temperature distribution at a larger $\mathrm{Gr}^{*}$ for both shear thinning and shear thickening fluids. On the other hand, at a given value of Ec, the impact of the nonDarcian parameter $\mathrm{Gr}^{*}$ is to increase the momentum and thermal boundary layer thickness for both shear thinning and shear thickening fluids.

The effects of viscosity/temperature parameter $\theta_{r}$ on the velocity $f^{\prime}(\eta)$ and temperature $\theta$ for both Darcy $\left(\mathrm{Gr}^{*}=0\right)$ and non-Darcy $\left(\mathrm{Gr}^{*}=2\right)$ flow cases are illustrated in Figures 5(a) and 6(a) for a shear thinning $(n=0.5)$ fluid 


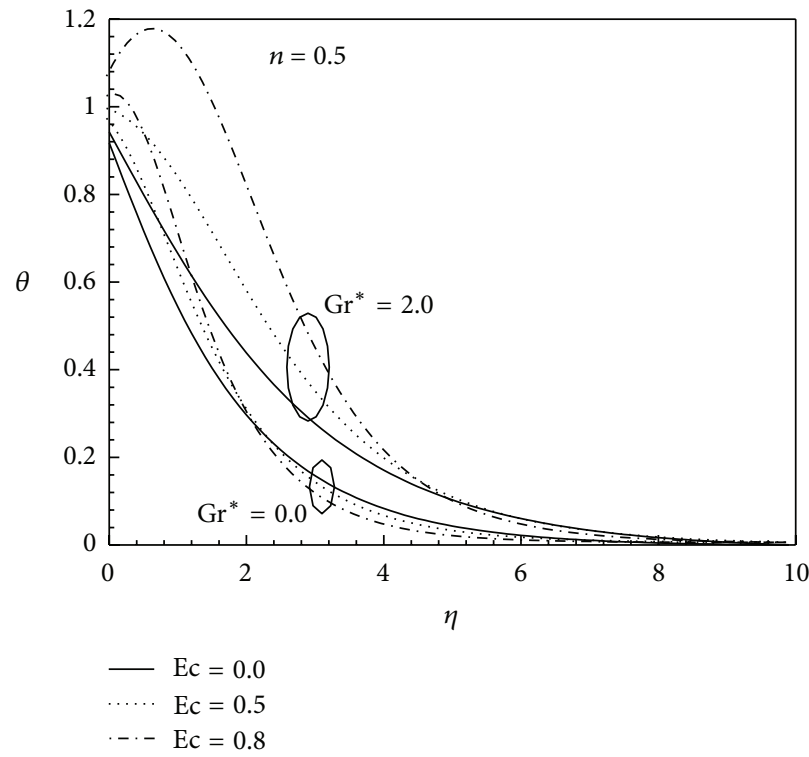

(a)

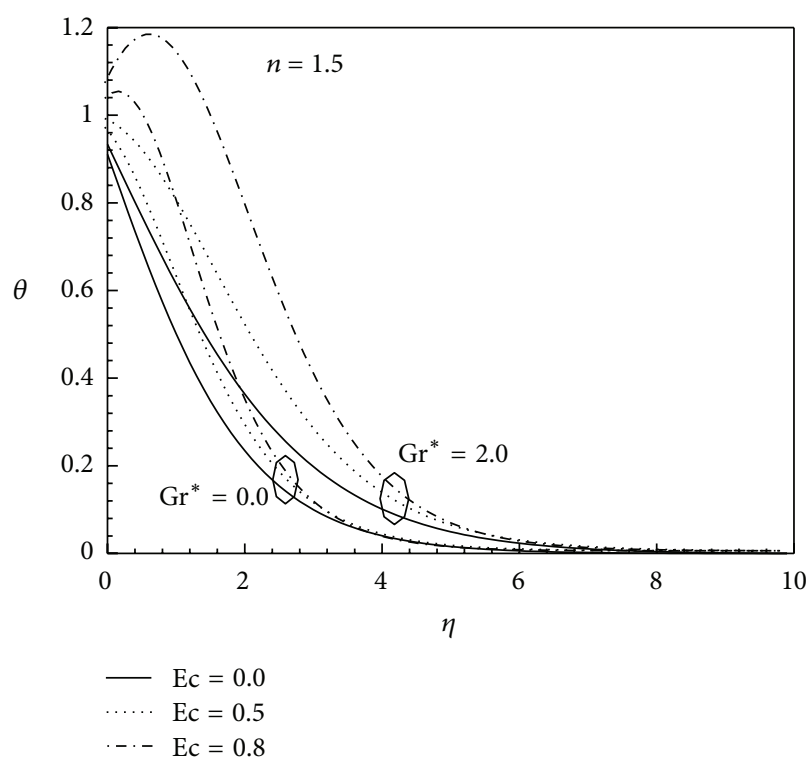

(b)

Figure 4: (a) Temperature profiles in the Darcy and non-Darcy cases for various values of Ec with $n=0.5, \theta_{r}=-1.0, M=0.2$, and $\Lambda=0.2$. (b) Temperature profiles in the Darcy and non-Darcy cases for various values of Ec with $n=1.5, \theta_{r}=-1.0, M=0.2$, and $\Lambda=0.2$.

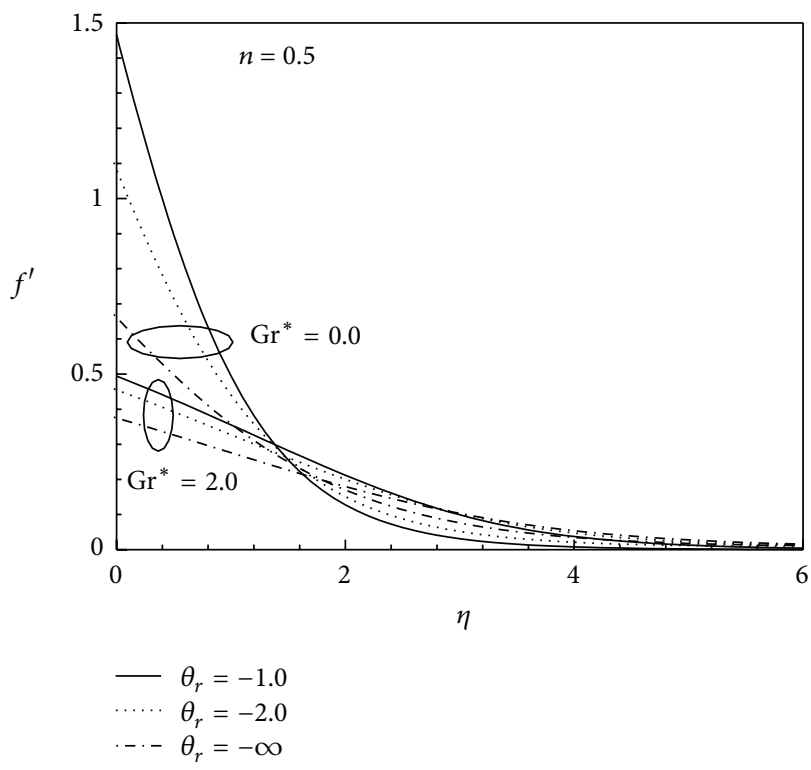

(a)

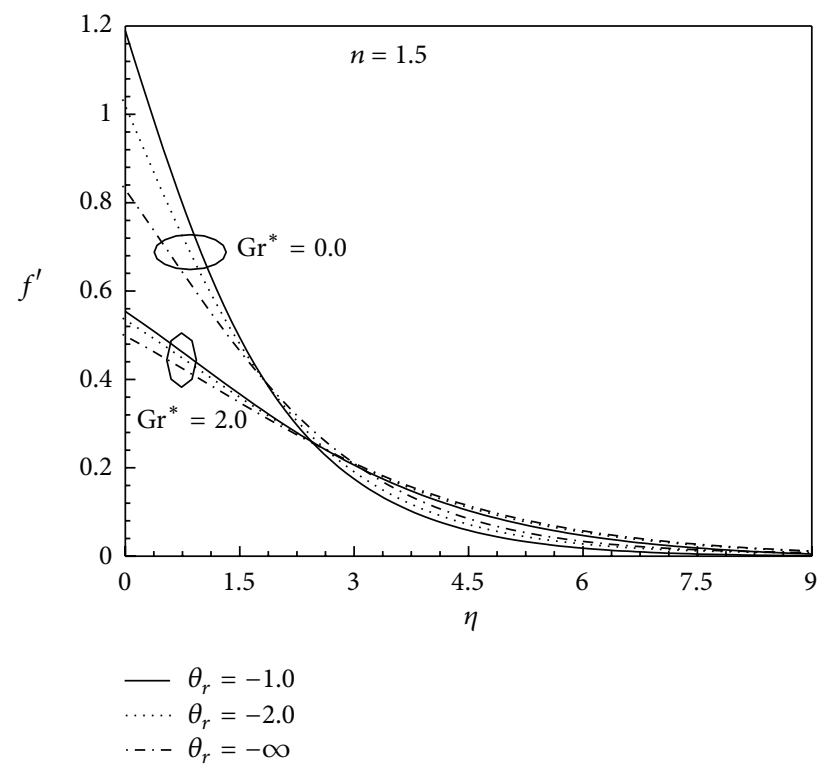

(b)

Figure 5: (a) Velocity profiles in the Darcy and non-Darcy cases for various values of $\theta_{r}$ with $n=0.5, M=0.2, \Lambda=0.2$, and Ec $=0.05$. (b). Velocity profiles in the Darcy and non-Darcy cases for various values of $\theta_{r}$ with $n=1.5, M=0.2, \Lambda=0.2$, and Ec $=0.05$.

and in Figures 5(b) and 6(b) for a shear thickening $(n=1.5)$ fluid. The results for the case of constant viscosity (i.e., $\theta_{r} \rightarrow$ $-\infty)$ are also shown in these figures. It is seen from these figures that the velocity and temperature distributions are significantly influenced by the value of $\theta_{r}$. It is observed from Figures 5(a) and 5(b) that the velocity $f^{\prime}(\eta)$ increases at a distance $\eta_{0}$ from the plate, where the value of $\eta_{0}$ depends on the values of $\mathrm{Gr}^{*}$ and $n$, while the exact opposite trend is noticed for $\eta>\eta_{0}$ as $\theta_{r}$ increases, and this conduct is veritable for all values of $\mathrm{Gr}^{*}$ and $n$. For example, $\eta_{0} \simeq 1.55$ for $\mathrm{Gr}^{*}=0$ and $n=0.5$, while the corresponding value of $\eta_{0} \simeq 1.8$ for $\mathrm{Gr}^{*}=0$ and $n=1.5$. For a specified value of $\mathrm{Gr}^{*}$, on the contrary, to increase $\theta_{r}$ is to reduce the temperature $\theta(\eta)$ for both shear thinning and shear thickening fluids as indicated in Figures 6(a) and 6(b). Comparison of Figures 5 and 6 shows that the effects of $\theta_{r}$ and $\mathrm{Gr}^{*}$ on 


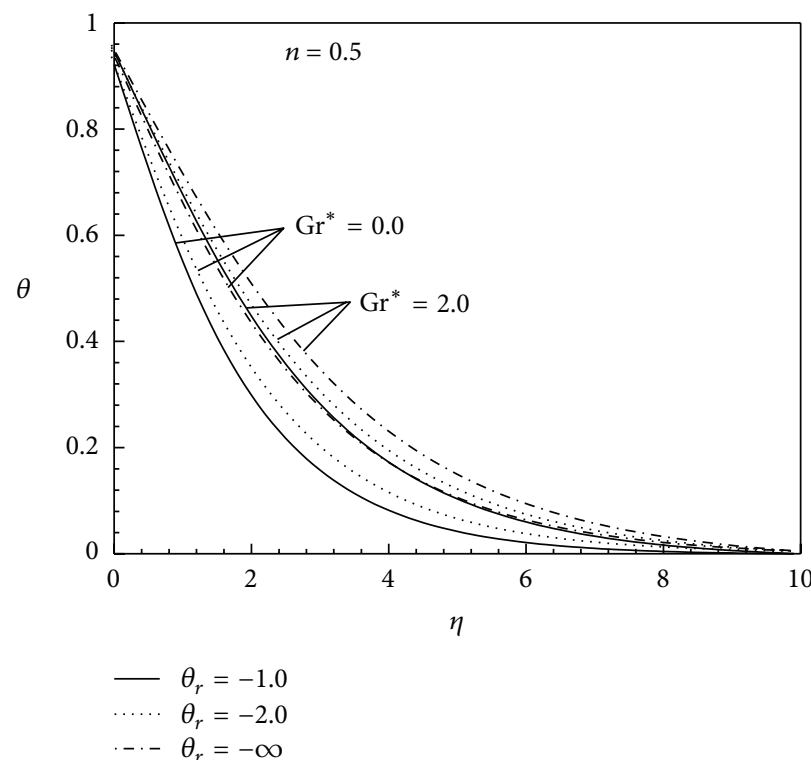

(a)

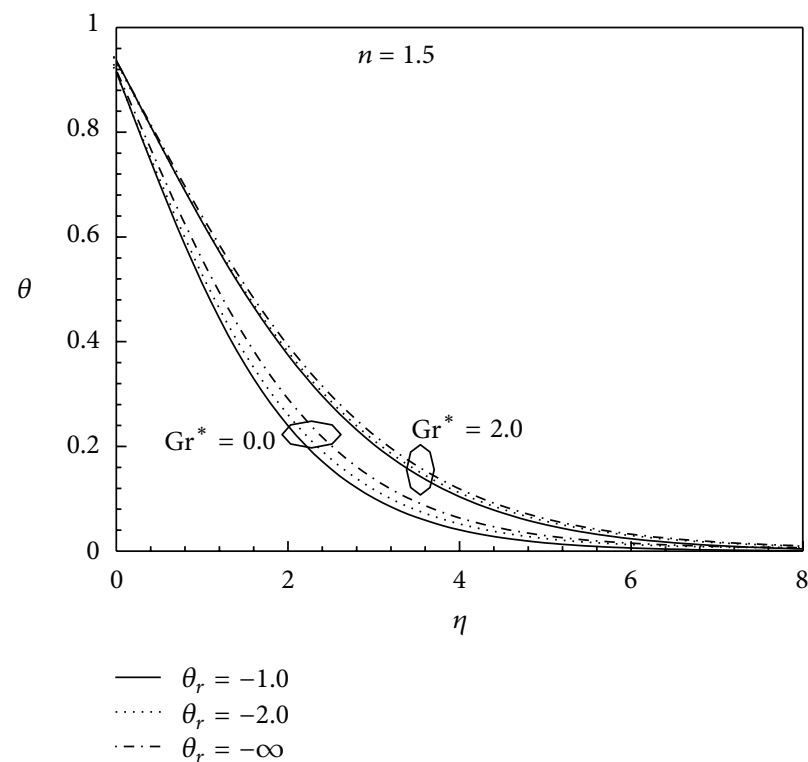

(b)

Figure 6: (a) Temperature profiles in the Darcy and non-Darcy cases for various values of $\theta_{r}$ with $n=0.5, M=0.2, \Lambda=0.2$, and Ec $=0.05$. (b) Temperature profiles in the Darcy and non-Darcy cases for various values of $\theta_{r}$ with $n=1.5, M=0.2, \Lambda=0.2$, and Ec $=0.05$.

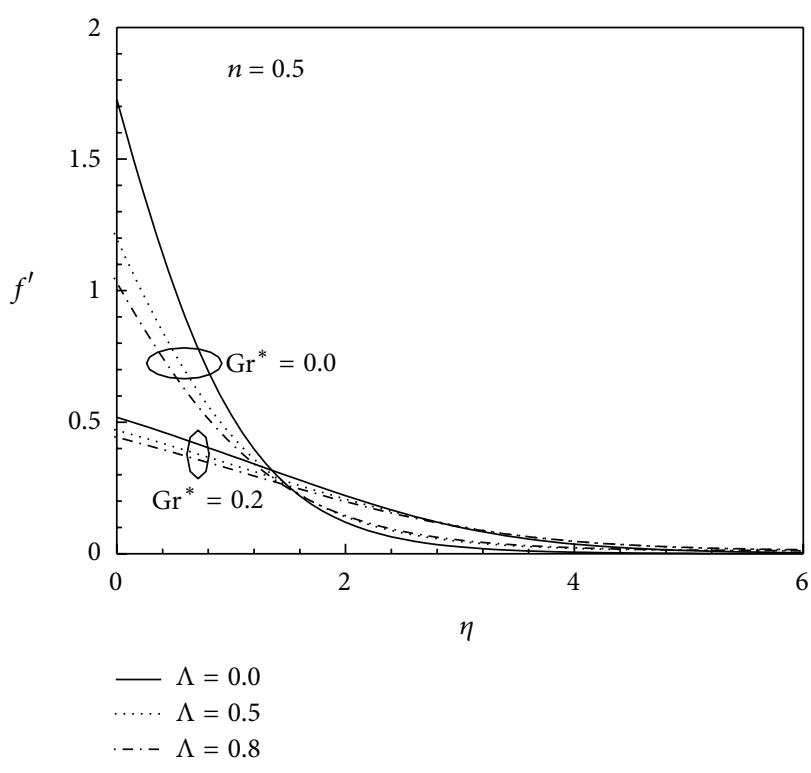

(a)

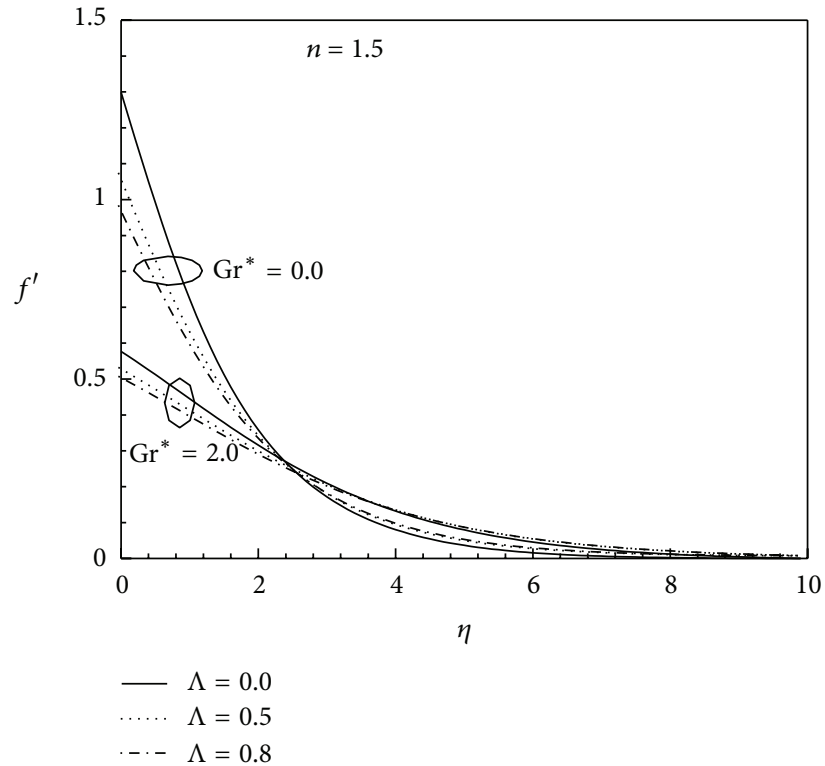

(b)

Figure 7: (a) Velocity profiles in the Darcy and non-Darcy cases for various values of $\Lambda$ with $n=0.5, \theta_{r}=-1.0, M=0.2$, and Ec $=0.05$. (b) Velocity profiles in the Darcy and non-Darcy cases for various values of $\Lambda$ with $n=1.5, \theta_{r}=-1.0, M=0.2$, and Ec $=0.05$.

the velocity and temperature profiles are found to be more intelligible for the case of shear thinning fluid.

Figures 7(a) and 8(a) demonstrate, respectively, the effects of temperature jump parameter $\Lambda$ on the dimensionless velocity and temperature profiles in the boundary layer for both Darcy and non-Darcy flow regimes for a shear thinning fluid, whereas Figures 7(b) and 8(b) are for a shear thickening fluid. For Darcy and non-Darcy flow cases, the velocity decreases rapidly near the boundary by increasing the temperature jump parameter $\Lambda$ as observed from Figures $7(\mathrm{a})$ and $7(\mathrm{~b})$. On the other hand, to increase the magnitude of temperature jump parameter $\Lambda$ is to reduce 


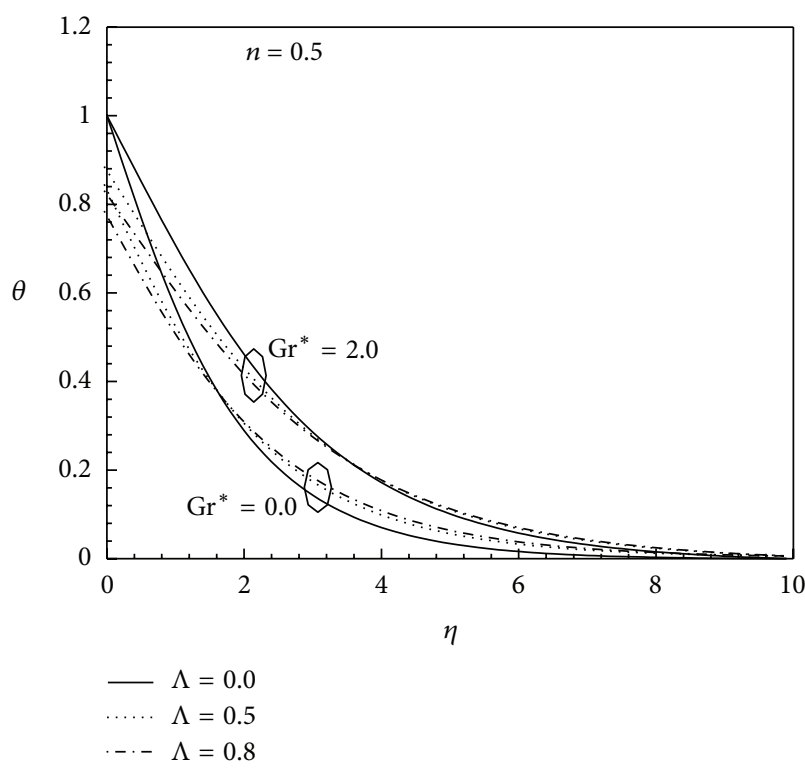

(a)

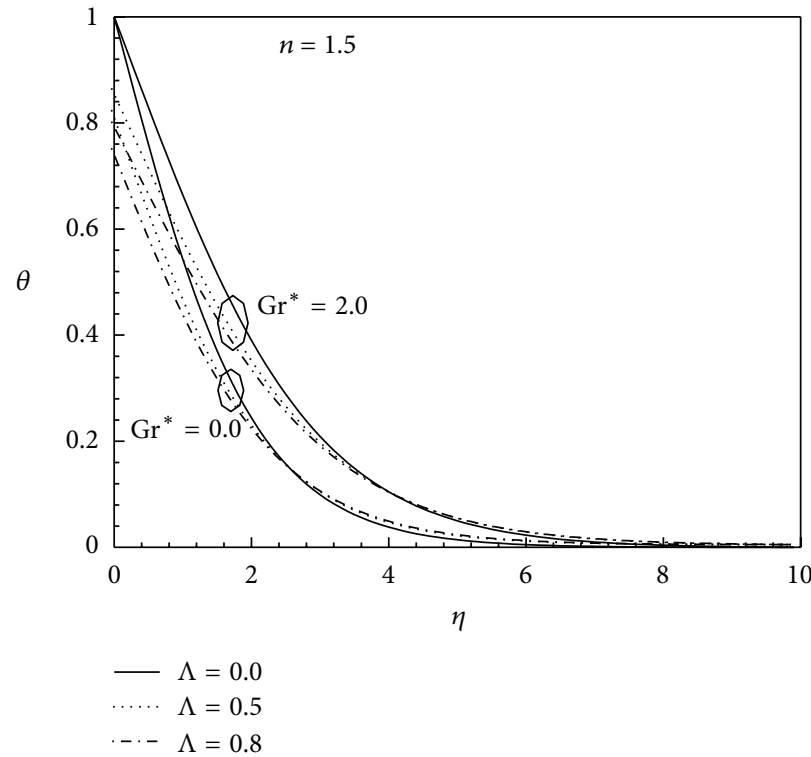

(b)

Figure 8: (a) Temperature profiles in the Darcy and non-Darcy cases for various values of $\Lambda$ with $n=0.5, \theta_{r}=-1.0, M=0.2$, and Ec $=0.05$. (b) Temperature profiles in the Darcy and non-Darcy cases for various values of $\Lambda$ with $n=1.5, \theta_{r}=-1.0, M=0.2$, and Ec $=0.05$.

TABLE 2: Variation of $f^{\prime}(0), \mathrm{Nu}_{x} \mathrm{Ra}_{x}^{-1 / 2}$, and $\theta(0)$ with $\theta_{r}, \Lambda, M$, and Ec at $n=0.5$.

\begin{tabular}{|c|c|c|c|c|c|c|c|c|c|}
\hline \multirow{2}{*}{$\theta_{r}$} & \multirow{2}{*}{$\Lambda$} & \multirow{2}{*}{$M$} & \multirow{2}{*}{$\mathrm{Ec}$} & \multicolumn{2}{|c|}{$f^{\prime}(0)$} & \multicolumn{2}{|c|}{$\mathrm{Nu}_{x} \mathrm{Ra}_{x}^{-1 / 2}$} & \multicolumn{2}{|c|}{$\theta(0)$} \\
\hline & & & & $\mathrm{Gr}^{*}=0$ & $\mathrm{Gr}^{*}=2$ & $\mathrm{Gr}^{*}=0$ & $\mathrm{Gr}^{*}=2$ & $\mathrm{Gr}^{*}=0$ & $\mathrm{Gr}^{*}=2$ \\
\hline-1.0 & 0.2 & 0.2 & 0.05 & 1.466160 & 0.494216 & 0.391782 & 0.270713 & 0.921644 & 0.945857 \\
\hline-2.0 & 0.2 & 0.2 & 0.05 & 1.094730 & 0.450206 & 0.343294 & 0.256483 & 0.931341 & 0.948703 \\
\hline$-\infty$ & 0.2 & 0.2 & 0.05 & 0.662177 & 0.369577 & 0.280718 & 0.233396 & 0.943856 & 0.953321 \\
\hline-1.0 & 0.0 & 0.2 & 0.05 & 1.727240 & 0.518504 & 0.463863 & 0.294485 & 1.000000 & 1.000000 \\
\hline-1.0 & 0.5 & 0.2 & 0.05 & 1.209750 & 0.462126 & 0.322324 & 0.241737 & 0.838838 & 0.879131 \\
\hline-1.0 & 0.8 & 0.2 & 0.05 & 1.039080 & 0.435250 & 0.276641 & 0.218961 & 0.778687 & 0.824831 \\
\hline-1.0 & 0.2 & 0.0 & 0.05 & 2.960420 & 0.533910 & 0.487462 & 0.279969 & 0.902508 & 0.944006 \\
\hline-1.0 & 0.2 & 1.0 & 0.05 & 0.561688 & 0.369883 & 0.27535 & 0.238824 & 0.944930 & 0.952235 \\
\hline-1.0 & 0.2 & 2.0 & 0.05 & 0.331220 & 0.272547 & 0.224427 & 0.210505 & 0.955115 & 0.957899 \\
\hline-1.0 & 0.2 & 2.0 & 0.0 & 1.453310 & 0.492309 & 0.411001 & 0.287924 & 0.917800 & 0.9424152 \\
\hline-1.0 & 0.2 & 2.0 & 0.5 & 1.616710 & 0.513857 & 0.161406 & 0.047102 & 0.967719 & 0.9905796 \\
\hline-1.0 & 0.2 & 2.0 & 0.8 & 1.78933 & 0.546404 & -0.094211 & -0.318840 & 1.018842 & 1.0637680 \\
\hline
\end{tabular}

the dimensionless temperature profile, and this behavior is conducted for both values of $\mathrm{Gr}^{*}$ as obvious from Figures 7(b) and 8(b). The temperature jump parameter $\Lambda$ demonstrates a more pronounced influence on the velocity and temperature distribution of a shear thinning fluid.

Representative numerical results for the fluid velocity at the plate $f^{\prime}(0)$, the local Nusselt number $\mathrm{Nu}_{x} \mathrm{Ra}_{x}^{-1 / 2}$, and the temperature of the plate $\theta(0)$ for various parametric values are presented in Table 2 for a shear thinning fluid with $n=0.5$ and in Table 3 for a shear thickening fluid with $n=1.5$. It can be seen from Table 2 that the fluid velocity at the plate $f^{\prime}(0)$ and the local Nusselt number $\mathrm{Nu}_{x} \mathrm{Ra}_{x}^{-1 / 2}$ increase while the temperature of the plate $\theta(0)$ decreases as the viscosity/temperature parameter $\theta_{r}$ increases. In contrast with $\theta_{r}$ effect, increases in the magnetic parameter $M$ result in decreases in the fluid velocity at the plate $f^{\prime}(0)$ and the local Nusselt number $\mathrm{Nu}_{x} \mathrm{Ra}_{x}^{-1 / 2}$, and increases in the temperature of the plate $\theta(0)$. Also, from Table 2 we observe that increases in the values of temperature jump parameter $\Lambda$ cause increases in the values of $f^{\prime}(0), \mathrm{Nu}_{x} \mathrm{Ra}_{x}^{-1 / 2}$ and $\theta(0)$. It can also be observed that as the Eckert number Ec increases, both the fluid velocity and the temperature at the plate $f^{\prime}(0)$ and $\theta(0)$, respectively, increase while the local Nusselt number $\mathrm{Nu}_{x} \mathrm{Ra}_{x}^{-1 / 2}$ decreases. Further, Table 2 shows that the behaviors of $f^{\prime}(0), \mathrm{Nu}_{x} \mathrm{Ra}_{x}^{-1 / 2}$, and $\theta(0)$ due to the effects of $\theta_{r}, \Lambda, M$, and Ec are the same for both Darcy and non-Darcy 
TABLE 3: Variation of $f^{\prime}(0), \mathrm{Nu}_{x} \mathrm{Ra}_{x}^{-1 / 2}$, and ; $\theta(0)$ with $\theta_{r}, \Lambda, M$, and Ec at $n=1.5$.

\begin{tabular}{lccccccccc}
\hline$\theta_{r}$ & $\Lambda$ & $M$ & $\mathrm{Ec}$ & $\mathrm{Gr}^{*}=0$ & $\mathrm{Gr}^{*}=2$ & $\mathrm{Gr}^{*}=0$ & $\mathrm{Nu}_{x} \mathrm{Ra}_{x}^{-1 / 2}$ & \multicolumn{2}{c}{$\theta(0)$} \\
\hline-1.0 & 0.2 & 0.2 & 0.05 & 1.189271 & 0.553978 & 0.424613 & 0.312988 & 0.915077 \\
-2.0 & 0.2 & 0.2 & 0.05 & 1.027950 & 0.532614 & 0.402245 & 0.307785 & 0.919551 & 0.937402 \\
$-\infty$ & 0.2 & 0.2 & 0.05 & 0.831943 & 0.495849 & 0.373951 & 0.299693 & 0.925210 & 0.940061 \\
\hline-1.0 & 0.0 & 0.2 & 0.05 & 1.298940 & 0.576869 & 0.483828 & 0.340723 & 1.000000 & 1.000000 \\
-1.0 & 0.5 & 0.2 & 0.05 & 1.067201 & 0.524817 & 0.361203 & 0.27957 & 0.819399 & 0.860215 \\
-1.0 & 0.8 & 0.2 & 0.05 & 0.976295 & 0.500298 & 0.315835 & 0.2531 & 0.747332 & 0.797520 \\
\hline-1.0 & 0.2 & 0.0 & 0.05 & 1.443571 & 0.591791 & 0.456514 & 0.321858 & 0.908697 & 0.935628 \\
-1.0 & 0.2 & 1.0 & 0.05 & 0.657822 & 0.429802 & 0.331357 & 0.279032 & 0.933729 & 0.944194 \\
-1.0 & 0.2 & 2.0 & 0.05 & 0.406623 & 0.323619 & 0.267707 & 0.244694 & 0.946459 & 0.951061 \\
\hline-1.0 & 0.2 & 2.0 & 0.0 & 1.183923 & 0.552565 & 0.445510 & 0.332016 & 0.910898 & 0.933597 \\
-1.0 & 0.2 & 2.0 & 0.5 & 1.255322 & 0.571986 & 0.168316 & 0.0675017 & 0.966336 & 0.986500 \\
-1.0 & 0.2 & 2.0 & 0.8 & 1.341190 & 0.60079 & -0.162245 & -0.336549 & 1.032449 & 1.067310 \\
\hline
\end{tabular}

flow cases, but these behaviors are more pronounced in the case of Darcy flow.

Values of $f^{\prime}(0), \mathrm{Nu}_{x} \mathrm{Ra}_{x}^{-1 / 2}$, and $\theta(0)$ in the case of a shear thickening fluid case of $n=1.5$ for the same physical parameters presented in Table 2 are listed in Table 3. Effects of all physical parameters on the $f^{\prime}(0), \mathrm{Nu}_{x} \mathrm{Ra}_{x}^{-1 / 2}$, and $\theta(0)$ are noticed to be qualitatively similar but with quantitatively increased or decreased magnitude as compared to the case of a shear thinning fluid. Comparison study of Tables 2 and 3 indicates for the Darcy case that $f^{\prime}(0)$ of the shear thinning fluid is higher than that of the shear thickening fluid for all values of $\theta_{r}, \Lambda, M$, and Ec while the opposite result was observed for the non-Darcy case $\left(\mathrm{Gr}^{*}=2\right)$. For the Darcy flow regime, Tables 2 and 3 show that the local Nusselt number $\mathrm{Nu}_{x} \mathrm{Ra}_{x}^{-1 / 2}$ of the shear thinning fluid is lower than that of the shear thickening fluid for all values of $\theta_{r}, \Lambda$, and Ec while the opposite trend is true for the non-Darcy flow regime. On the contrary, the magnetic field $M$ has an opposite effect to the effects of $\theta_{r}, \Lambda$, and Ec on the $\mathrm{Nu}_{x} \mathrm{Ra}_{x}^{-1 / 2}$ for both Darcy and non-Darcy flow cases. On the other hand, it is observed from these tables that the temperature of the plate $\theta(0)$ for a shear thinning fluid is higher than that for a shear thickening fluid for all values of $\theta_{r}, \Lambda$, and $M$, and this is true for both Darcy and non-Darcy flow regimes. Finally, keeping all other parameters fixed, it is shown from Tables 2 and 3 that due to the transition from Darcy regime to non-Darcy regime, the fluid velocity at the plate $f^{\prime}(0)$ and the local Nusselt number $\mathrm{Nu}_{x} \mathrm{Ra}_{x}^{-1 / 2}$ decrease while the plate temperature $\theta(0)$ increases.

\section{Conclusion}

The problem of MHD free-convective flow and heat transfer from a vertical flat plate embedded in a non-Darcian porous medium saturated with a power law non-Newtonian fluid is investigated with viscous dissipation variable viscosity. By using similarity transformations, the governing boundary layer equations, for momentum and thermal energy are reduced to coupled non-linear ordinary differential equations which are then solved numerically using a shooting method. The results pertaining to the present study indicate that the flow and the temperature field are significantly influenced by considering the non-Darcy case for the pseudoplastic and dilatant fluids. Increasing the thermal slip parameter causes decreasing the velocity and temperature for Darcy and nonDarcy flow cases. Also increasing the thermal slip parameter causes reducing the heat transfer from the sheet.

\section{Acknowledgments}

This research is supported by King Khalid University (KKU), Saudi Arabia, under the Project no. KKU-SCI-11-12. This support is highly appreciated and acknowledged. Also the authors are very thankful to the referees for their encouraging comments and constructive suggestions to improve the presentation of this paper.

\section{References}

[1] P. Cheng and C. T. Hsu, "Higher-order approximations for Darcian free convective flow about a semi-infinite vertical flat plate," Journal of Heat Transfer, vol. 106, no. 1, pp. 143-151, 1984.

[2] R. M. Fand and J. Brucker, "A correlation for heat transfer by natural convection from horizontal cylinders that accounts for viscous dissipation," International Journal of Heat and Mass Transfer, vol. 26, no. 5, pp. 709-716, 1983.

[3] R. M. Fand, T. E. Steinberger, and P. Cheng, "Natural convection heat transfer from a horizontal cylinder embedded in a porous medium," International Journal of Heat and Mass Transfer, vol. 29, no. 1, pp. 119-133, 1986.

[4] A. Bejan, Convection Heat Transfer, Wiley, New York, NY, USA, 1984.

[5] C. L. Tucker III and R. B. Dessenberger, "Governing equations for flow and heat transfer in stationary fiber beds," in Flow and Rheology in Polymer Composites Manufacturing, S. G. Advani, Ed., Elsevier Science, Amsterdam, The Netherlands, 1994.

[6] F. S. Ibrahim, M. A. Mansour, and S. M. Abdel-Gaied, "Radiative and thermal dispersion effects on non-Darcy natural convection with lateral mass flux for non-Newtonian fluid from a 
vertical flat plate in a saturated porous medium," Transport in Porous Media, vol. 61, no. 1, pp. 45-57, 2005.

[7] M. F. El-Amin, "Combined effect of magnetic field and viscous dissipation on a power-law fluid over plate with variable surface heat flux embedded in a porous medium," Journal of Magnetism and Magnetic Materials, vol. 261, no. 1-2, pp. 228-237, 2003.

[8] M. A. Mansour and N. A. El-Shaer, "Effect of magnetic field on non-Darcy axisymmetric free convection in a power-law fluid saturated porous medium with variable permeability," Journal of Magnetism and Magnetic Materials, vol. 250, pp. 57-64, 2002.

[9] A. Mahdy, "Soret and Dufour effect on double diffusion mixed convection from a vertical surface in a porous medium saturated with a non-Newtonian fluid," Journal of Non-Newtonian Fluid Mechanics, vol. 165, no. 11-12, pp. 568-575, 2010.

[10] S. Jayanthi and M. Kumari, "Effect of variable viscosity on non-Darcy free or mixed convection flow on a vertical surface in a non-Newtonian fluid saturated porous medium," Applied Mathematics and Computation, vol. 186, no. 2, pp. 1643-1659, 2007.

[11] A. M. Salem, "Variable viscosity and thermal conductivity effects on MHD flow and heat transfer in viscoelastic fluid over a stretching sheet," Physics Letters A, vol. 369, no. 4, pp. 315-322, 2007.

[12] A. V. Shenoy, "Darcy-Forchheimer natural, forced and mixed convection heat transfer in non-Newtonian power-law fluidsaturated porous media," Transport in Porous Media, vol. 11, no. 3, pp. 219-241, 1993.

[13] R. H. Christopher and S. Middleman, "Power-law flow through a packed tube," Industrial and Engineering Chemistry Fundamentals, vol. 4, no. 4, pp. 422-426, 1965.

[14] R. V. Dharmadhikari and D. D. Kale, "Flow of non-Newtonian fluids through porous media," Chemical Engineering Science, vol. 40, no. 3, pp. 527-528, 1985.

[15] M. Kumari, "Effect of variable viscosity on non-Darcy free or mixed convection flow on a horizontal surface in a saturated porous medium," International Communications in Heat and Mass Transfer, vol. 28, no. 5, pp. 723-732, 2001.

[16] F. C. Lai and F. A. Kulacki, "The effect of variable viscosity on convective heat transfer along a vertical surface in a saturated porous medium," International Journal of Heat and Mass Transfer, vol. 33, no. 5, pp. 1028-1031, 1990.

[17] T. Y. Na, Computational Methods in Engineering Boundary Value Problems, vol. 145 of Mathematics in Science and Engineering, Academic Press, New York, NY, USA, 1979. 


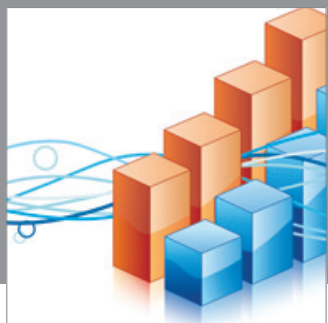

Advances in

Operations Research

mansans

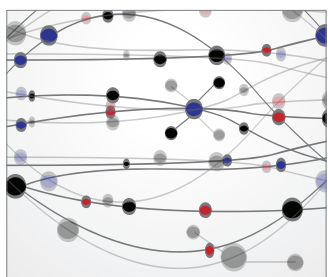

The Scientific World Journal
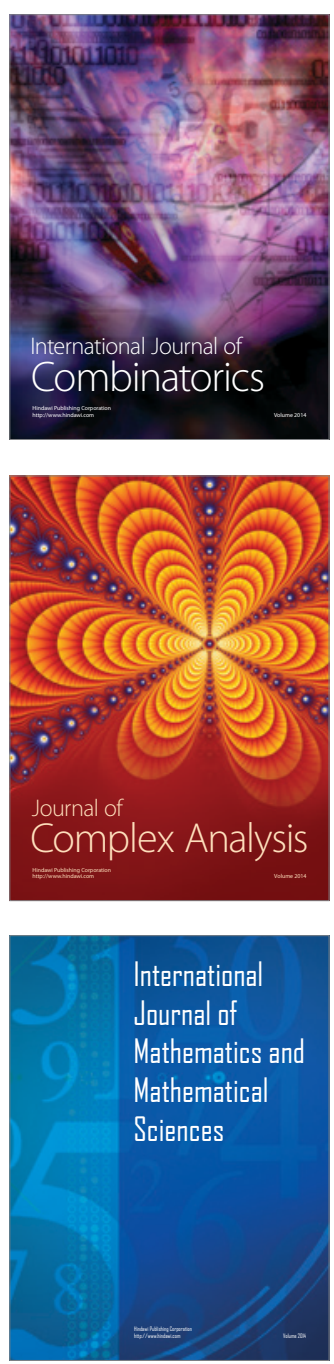
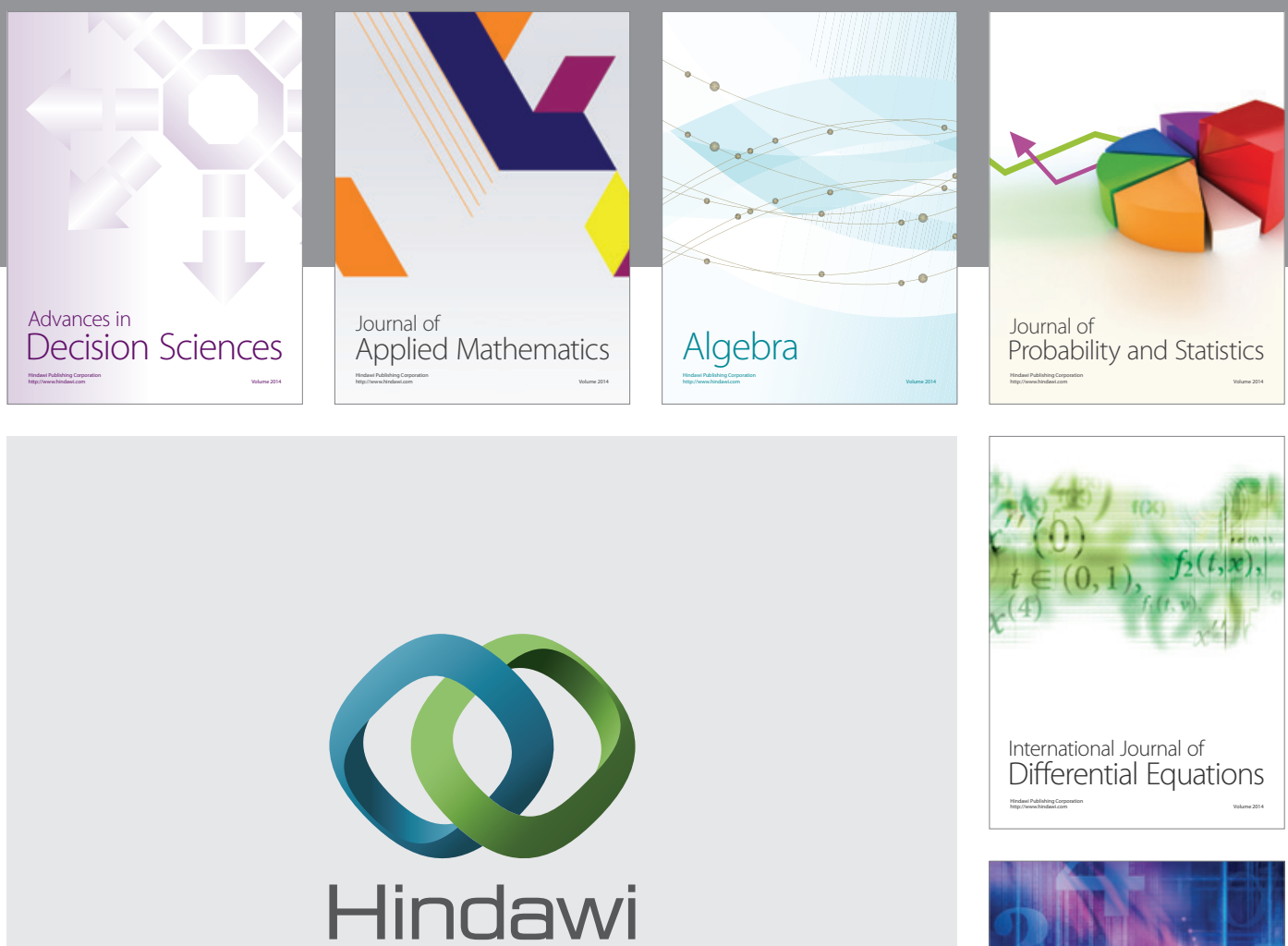

Submit your manuscripts at http://www.hindawi.com
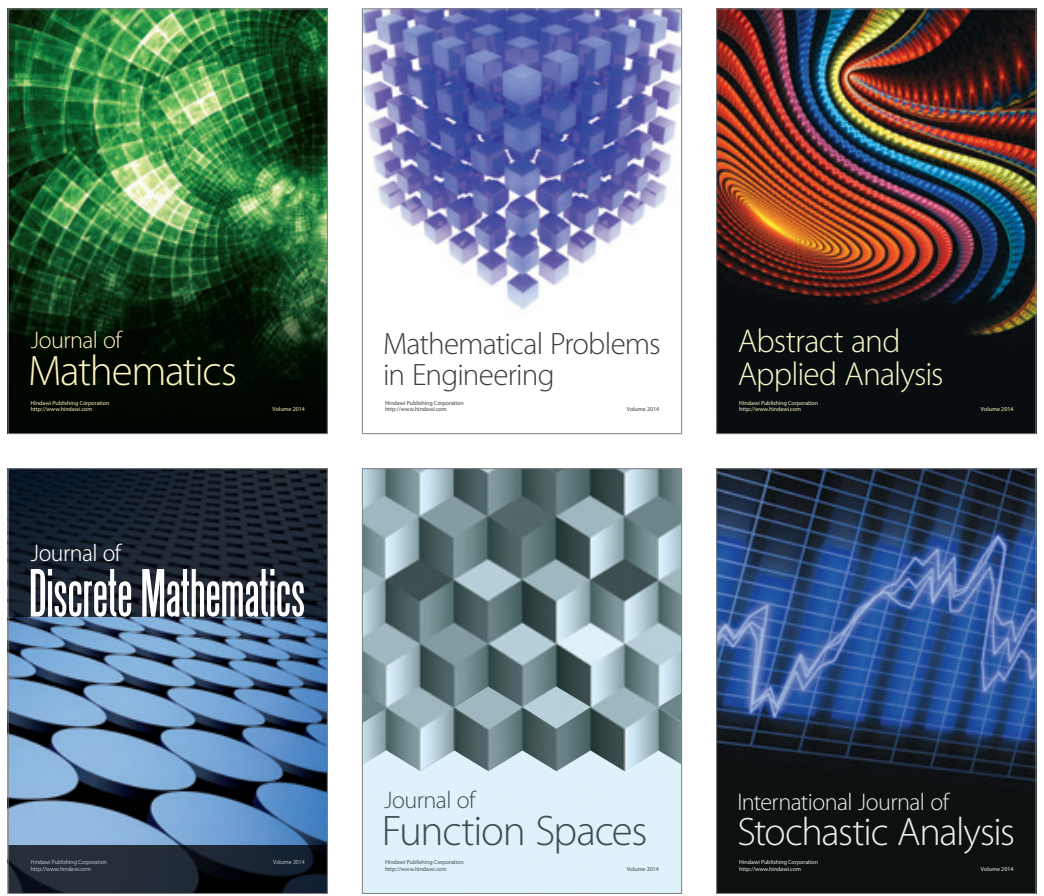

Journal of

Function Spaces

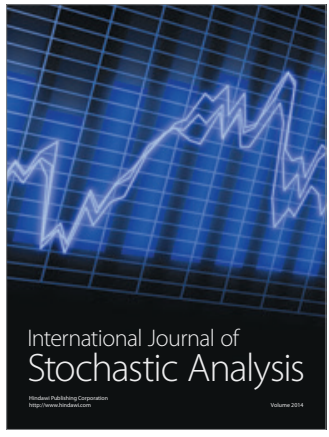

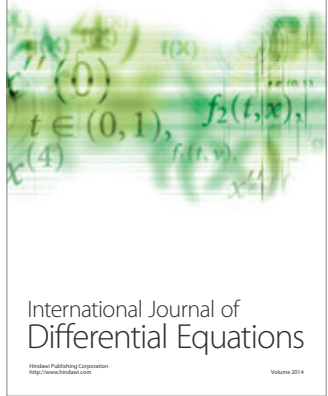
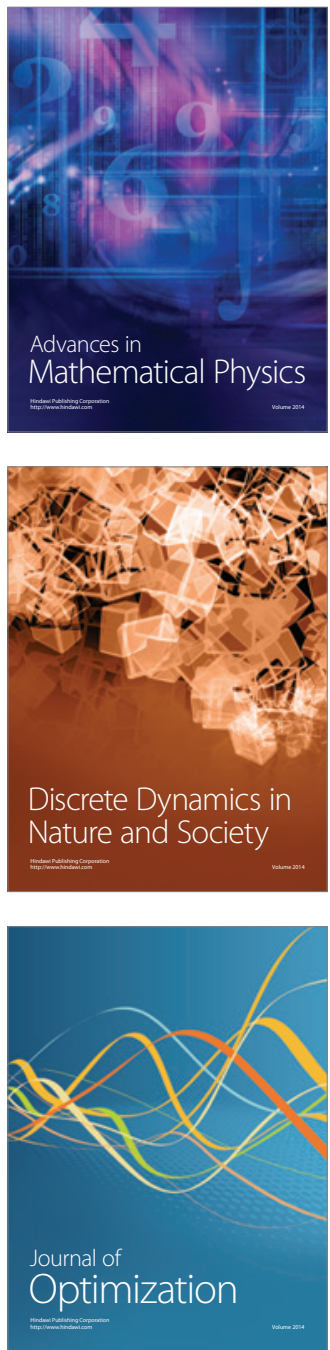